\title{
Exploration of the Optimization of the Use Efficiency of the Automatic Dispenser in Outpatient Pharmacy
}

\author{
Zhong Huiting, Zhang Zhidong* \\ Department of Pharmacy, The First Affiliated Hospital of Jinan University, Guangzhou, China \\ Email address: \\ hinsabc@163.com (Zhong Huiting), tzzd0608@jnu.edu.cn (Zhang Zhidong) \\ ${ }^{*}$ Corresponding author
}

To cite this article:

Zhong Huiting, Zhang Zhidong. Exploration of the Optimization of the Use Efficiency of the Automatic Dispenser in Outpatient Pharmacy. Science Journal of Clinical Medicine. Vol. 10, No. 1, 2021, pp. 12-15. doi: 10.11648/j.sjcm.20211001.13

Received: February 23, 2021; Accepted: March 17, 2021; Published: March 30, 2021

\begin{abstract}
Objective: With the advent of pharmacy automation system, pharmacists can liberate themselves from the heavy work of dispensing drugs and devote more time to the professional service of pharmacy. To improve the efficiency of dispensing machine in outpatient pharmacy and reduce the frequency of machine malfunction maintenance. Method: Through observation and detailed records of 645 occurrences of CONSIS-H5 dispenser failures from September 18 to September 24, 2017 and October 9 to October 22, 2017, research and analysis of the application process optimization plan of the dispenser. Result: Through the use of various management methods to propose improvement measures and maintenance methods, the failure maintenance rate of the dispenser has been reduced from the original average of 30.7 times per day to an average of 15.3 times per day, a decrease of $43.65 \%$. Conclusion: The CONSIS-H5 medicine dispenser has undergone a series of targeted adjustments and optimizations, reducing the failure rate and reducing the work intensity of the staff. At the same time, the efficiency improvement greatly reduces the waiting time of patients, which improves the patient's satisfaction with the hospital and improves the quality of pharmacy services.
\end{abstract}

Keywords: Outpatient Pharmacy, CONSIS-H5 Dispenser, Efficiency Optimization

\section{Introduction}

With society's development, the volume of business has increased rapidly by introducing new technology and emphasis on the medical industry. As the patient's final station in the hospital, the outpatient pharmacy must adapt to the times' development. Get rid of outmoded and inefficiency manual mode. It admits of no delay to further improve pharmaceutical care to carry out the drug collection work orderly [1-3]. Therefore, our hospital brings in two CONSIS-H5 dispensers from Willach (Shanghai) Pharmacy Solutions $\mathrm{GmbH}$ in November 2016. The dispensers improve medicine distribution efficiency, reduces the pharmacist's dispensing error and labour intensity, and shortens patients' waiting time. However, the dispensers setup and hardware also appear some problems like medicine jamming after a period of use [4]. Therefore it is essential to optimize the dispenser's operational plan according to its functional characteristics and needs $[5,6]$.

\section{Materials and Methods}

Our hospital, also known as Guangzhou Overseas Chinese Hospital or the First Clinical Medical College of Jinan University, is an affiliated hospital of Jinan University, which is the national "211 Project" of key comprehensive university directly under the guidance of Overseas Chinese Affairs Office of State Council. It is also an AAA public general hospital for medical treatment, medical training, scientific research, disease prevention, health care, and rehabilitation service. The average daily prescription quantity of the outpatient department was 4200.

Equip outpatient pharmacies with two Weller Heights CONSIS-H5 dispensers according to the hospital's outpatient volume and development needs. Each dispenser has two central engine units, four spiral straight hair outlet, nine pre-prepared medicine outlets and four sets of intelligent medicine cabinets. The host dosing unit can store about 20000 boxes of medicines. A total of eight dosing tracks in dosing 
area, four for bulk dosing track and four for general dosing rail. In this paper, CONSIS-H5 dispenser is taken as the research object, and its efficiency optimization scheme is discussed and analyzed.

\subsection{Data Collection}

The dispenser's failure from September 18 to September and October 9 to October 22, were collected by pharmacists on duty at the western pharmacy as per person per shift. Relevant parameters and failure process are registered in detail. Registration time is 24 hours a day.

\subsection{Method of Data Capture}

All the window pharmacists in the Western Pharmacy shall record the dispenser's malfunctions according to each person in each shift. The records divided into two parts, dosing module and dosing module failure. The records include occurrence time, track, event description, specific drug name and remarks [7-9].

\section{Results}

In this inspection, there were 645 failures in 21 days, with an average of 30.7 failures per day. The fault data are classified and counted after the data mining and analysis again. The fault data are classified and counted again and draw the Plato. By the $80 / 20$ rules, the critical points of improvement are drug delivery system's jamming fault and the manipulator's drug jamming fault.

Table 1. The template of fault Events Log Sheet. Date: Registrant.

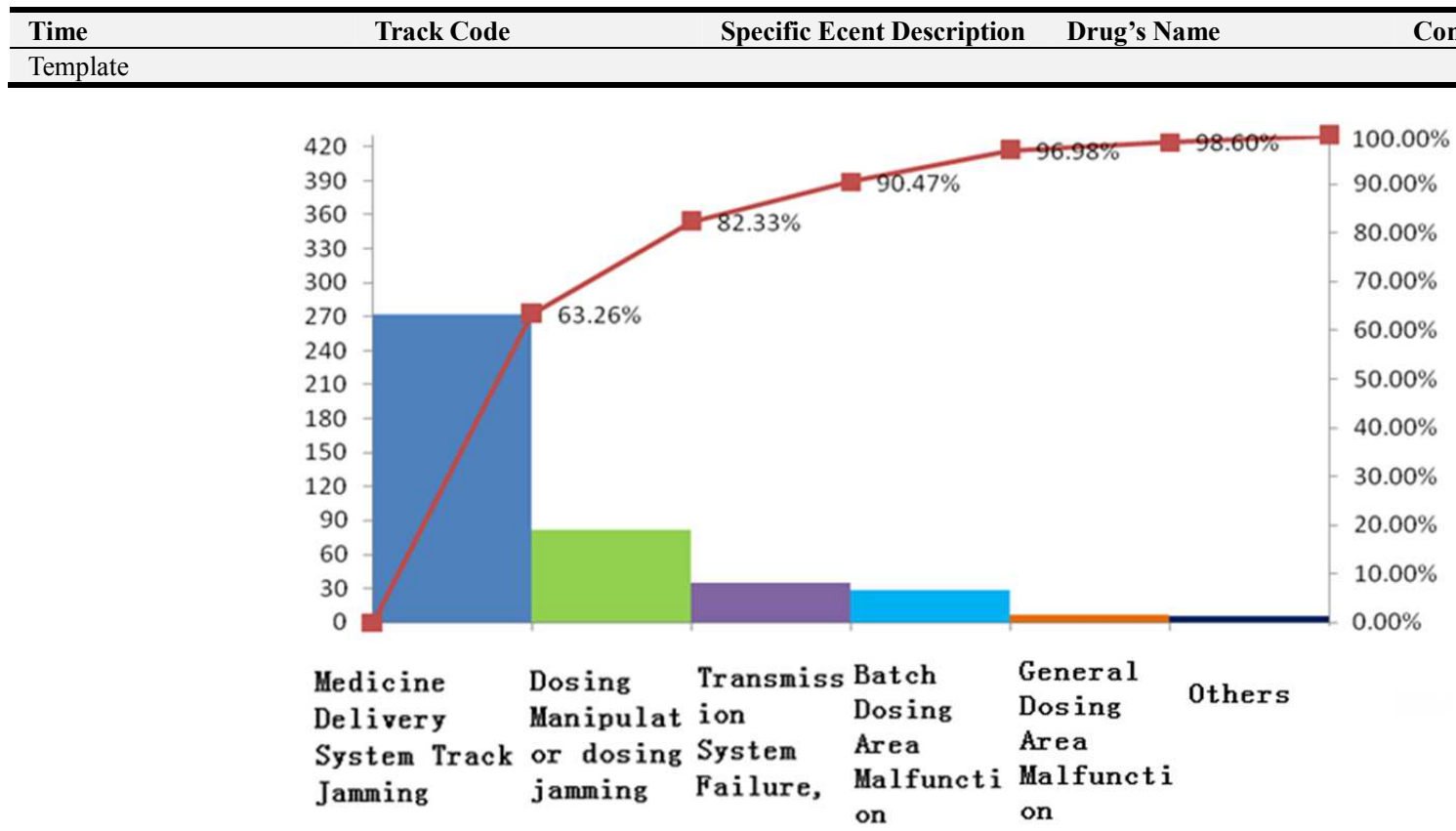

Figure 1. The number of failures of automatic dispensing machine is Platonic before efficiency optimization.

\subsection{Data Analysis}

Combined with the recorded data and the results drawn by Plato, the improvement focuses on the drug jamming of the track drug delivery system and the mechanical hand's drug jamming. All the team members "brainstorm" the main reasons for the failure of the dispenser. It concluded that the main reasons for drug jam in the orbital drug delivery system are shrapnel fault (machine) and lack of drug delivery standards (system). The main reasons for manipulator dosing jam are no inventory before dosing, track bulkhead displacement and lack of dosing operation norms.

\subsection{Optimization Countermeasures}

According to the analysis of the cause of the card medicine, the corresponding optimization countermeasures formulated. Implement and record, observe the effectiveness of each strategy.

\subsubsection{Optimization Strategy I: Rationally Adjust the Drug Entering Machine and Drug Discharging Mode}

It mainly aimed at drug jam in the track drug delivery system. The overweight or light drug will affect drug delivery accuracy, which is easy to cause drug errors.

Measures: 1, overweight or light drugs in the machine will be eliminated. Pills with complete packaging less than $10 \mathrm{~g}$ and more than $80 \mathrm{~g}$ per box will not add to the engine. 2, eliminate the drugs with a low utilization rate. Take the drug delivery data in recent two months, and the drugs with fewer than 20 boxes shall be removed from the machine's drug delivery range. 3 , the original shrapnel counting drug delivery was replaced with single track double shrapnel delivery for drugs with high drug delivery error rate known from record statistics.

Effect for Measures: One week after the implementation of countermeasure 1 , the number of drug jams in the orbital drug 
delivery system dropped from 19.4 times a day to 11.7 times a day, a decrease of $39.69 \%$.

\subsubsection{Optimization Strategy II: Formulate a Unified Standard System for Drug Addition and Troubleshooting}

Mainly for the manipulator dosing jam, dosing operation is not standard, the ability to discharge the fault is low with not standard. Lack of troubleshooting operation norms, some personnel lack troubleshooting skills and awareness, easy to cause the machine's chain jam.

Measures: 1, joint with the machine manufacturer's engineers to develop the troubleshooting norms of drug jamming, organize the whole team for on-site training in batches, and arrange special personnel to track and guide each employee to be familiar with troubleshooting operations for 14 days. 2, the unified setting of the number and time of machine counting every day.

Effect for Measures: After a week of inspection, after implementing Strategy II, The Times of mechanical hand dosing jamming decreased from 15.8 times per day to 10.20 times, a decrease of $35.44 \%$.

\subsubsection{Strengthen Problem Awareness, and Regularly Maintain the Problems Within the Specified Time}

Due to the lack of unified record and regular maintenance of problems encountered at ordinary times, the problems were not solved quickly.

Measures: 1, set up a machine fault and problem logbook for problems/faults encountered. 2, arrange regular staff and engineers to maintain and clean the machine and report any problematic problems.

Effect for Measures: The total frequency of drug jam decreased from 30.7 times per day to 17.3 times per day, decrease for $43.65 \%$.

\subsection{Optimistic Effect}

\subsubsection{The Speed of Dispensing Prescription Is Accelerated}

The average waiting time of patients is shortened, that is, the time from seeing the name on the screen to getting the medicine is shortened.

Table 2. Comparison of waiting time of patients before and after optimization.

\begin{tabular}{llll}
\hline Average Wait Time & Before Optimization & After Optimization & Magnitude of Improvement \\
\hline Whole Day (8:00-12:00; 14:30-17:30) Average Time & $407 \mathrm{~s}$ & $303 \mathrm{~s}$ & $25.56 \%$ \\
\hline
\end{tabular}

\subsubsection{Error Reduction}

It can be seen from the inspection that the cases of drug leakage or excessive drug release on track decreased from 19.4 times per day to 11.7 times per day.

\section{Discuss}

As the last line of defense for patients' medical behavior in outpatient western pharmacies, it is related to the medication safety of outpatients, and it is also an important part of the image of the hospital [11].

Since our hospital was equipped with the CONSIS-H5 automatic medicine dispenser, it had significantly reduced the waiting time of patients in the queue, improved the satisfaction of patients with the pharmacy, and reduced the labor intensity and the number of errors of the pharmacist, but there was still much room for improvement and improvement [12].

This efficiency optimization and improvement activity used the relevant steps of quality control tools, analyzed the reasons that may lead to the failure of the automatic dispensing machine through brainstorming, and formulated various improvement plans and measures for these reasons, further reducing the waiting time of patients and reducing the medicines [13]. The allocation error rate improves the dispensing accuracy and efficiency of the dispensing machine. The optimal configuration of dispensing machine and reasonable program setting are closely related to the working efficiency of pharmacy. How to further improve the efficiency of dispensing machine is the key to upgrade the level of pharmaceutical care in hospital pharmacy from traditional dispensing mode to pre-prescription review and standardized guidance for patients' medication, which is also the direction of our pharmacy's efforts to improve self-value [14]. Therefore, through the observation and record of the dispensing machine at the present stage, this paper makes a series of improvement plans, and successfully optimises the efficiency of the dispensing machine. But there was still lack of imperfections, such as caused by a lack of understanding of the dispensing machine software program, failed to timely handle the dispensing machine software error, lead to the failure time is long and restricted by routine work in the field and so on, not the other problems to be resolved for further optimization, such as we will see this as the outpatient pharmacy next focus on the task in the project optimization.

\section{Conclusion}

In general, we should establish the awareness of problems in work, keep detailed records of various problems, summarize experience regularly, and make optimization and adjustment for problems in work. Further optimize various work processes, reduce dispensing errors, improve patient satisfaction, and further improve the level of pharmaceutical care.

\section{References}

[1] Tieshan W, Suiqiong W, Shaoxiong Z, Mingjin C and Rui F. (2020). Construction and Practice of Automatic Drug Delivery System in Outpatient Pharmacy of Our Hospital. China Pharmacy 31: 2415-2421. 
[2] Fu-yan Z. (2020). Analysis of the Effect of Using Quality Control Circle to Reduce the Error Rate of Automatic Drug Pendulum. China Health Industry 17: 49-50, 53.

[3] Hua X, Mei-li Z and Bao-qin X. (2020). Application of quality control circle in reducing the number of accidents in automatic dispensing machine in outpatient pharmacy. China Modern Medicine 27: 175-178.

[4] Yakun Z, Liangliang H, Yiling H and Yan F. (2020). Practice of optimizing the workflow of outpatient automated pharmacy. Pharmaceutical Care and Research 20: 70-72.

[5] Menglin W, Dahai D, Dengli Z and Yongqing W. (2019). Analysis of the Effect and Problem of the Automatic Medicine Dispenser Used on a Large Scale in Outpatient Pharmacy. Modern Hospital: 518-521.

[6] Jia-ling H, Wen-yan Y, Ru-qing Y, Xiao-yun L and ZHi-hui L. (2019). Problems and countermeasures found in the use of automated dispensing systems. China Modern Medicine 26: 144-146.

[7] Zhiran H, Pengyu Z, Zijuan W, Jing S and Yuanli L. (2018). Multivariate analysis of influencing factors of outpatients'satisfaction in hospitals of different types. Chinese Journal of Hospital Administration 34: 93-98.

[8] Zhifang N. (2018). Analysis of problems found in the use of rapid dispensing system in outpatient pharmacy. Modern Medicine and Health Research: 60.
[9] Qingcheng Z. (2018). The optimization practice of the work flow of the automatic dispensing machine in the outpatient pharmacy of our hospital. Strait Pharmaceutical Journal 30: 293-294.

[10] Le Z and Xueyu L. (2018). Analysis of medicine delivery errors in automated pharmacy quick delivery system. Strait Pharmaceutical Journal 30: 291-293.

[11] Maoben L, Yong X, Lijuan C, Yuemei Z and Dongming G. (2017). A survey of patient satisfaction in outpatient pharmacy under the current automated dispensing mode. Chinese Medicine of Factory and Mine: 287-288.

[12] Limei H, Zhiyuan Y, Lili Z and Aiwen H. (2017). Practice of quality control circle to reduce failure event times of automatic drug dispensing machine in outpatient pharmacy. Anhui Medical and Pharmaceutical Journal 21: 2129-2132.

[13] Zhijie L, Jiwei C, Miaofa Y, Zheng Z and Rui Z. (2016). Establishment and Continuous Quality Improvement of Outpatient Pharmacy Work Pattern Based on the Application of the Automatic Drug Dispensing Machine. China Pharmacist 19: 1770-1772.

[14] Hui L, Rong C, Dengke W and Jihong G. (2014). Features of automated outpatient pharmacy in our hospital. Pharmaceutical and Clinical Research 2: 193-194. 